\title{
ERP-Systeme in der Lehre - ein vergleichendes, hochschulübergreifendes Seminar mit mittelgroßen ERP-Systemen
}

\author{
Axel Winkelmann', Christian Leyh², Norbert Frick' \\ ${ }^{1}$ Institut für Wirtschafts- und Verwaltungsinformatik, \\ Professur für Betriebliche Anwendungssysteme, \\ Universität Koblen₹-Landau \\ ${ }^{2}$ Lehrstuhl für Wirtschaftsinformatik, \\ insh. Informationssysteme in Industrie und Handel, \\ Technische Universität Dresden
}

\section{Motivation}

Standardisierte Enterprise Resource Planning (ERP) - Systeme sind heutzutage in einem Großteil der Unternehmen im Einsatz. Zum Beispiel setzen über $92 \%$ aller deutschen Industrieunternehmen ERP-Systeme ein (Konradin 2009, S. 49). Aufgrund dieser starken Nachfrage existieren viele unterschiedliche ERP-Systeme mit unterschiedlichen Technologien und Philosophien, so dass der Markt vor allem im Bereich der klein- und mittelständischen Unternehmen (KMU) sehr stark fragmentiert ist (Winkelmann et al. 2007). Diese Vielzahl von Herstellern und Systemen erschwert es den Anwender-Unternehmen, die „richtige“ Software zu finden und für das von ihnen ausgewählte System entsprechende Fachkräfte an sich zu binden. Auch für zukünftige Investitionsentscheidungen bezüglich des Einsatzes, des Upgrades oder der Umstellung von ERP-Systemen ist es wichtig, über entsprechendes Fachwissen und Kompetenzen im Unternehmen zu verfügen (Becker et al. 2007). Dies ist essentiell, da Fehler im Bereich der Auswahl, der Einführung oder des Betriebes von ERP-Systemen finanzielle Nachteile nach sich ziehen, die bis zu den Insolvenzen der betroffenen Unternehmen führen können. Beispiele derartiger Negativ-Szenarien lassen sich in der Literatur finden (Barker und Frolick 2003; Hsu et al. 2006). Um dem vorzubeugen, ergibt sich die Notwendigkeit für Hochschulen, ihren Studierenden und späteren Absolventen das geforderte Fachwissen, insbesondere in informationssystembezogenen Studiengängen wie beispielsweise dem Studiengang Wirtschaftsinformatik, zu vermitteln (Venkatesh 2008, S. 141-142). 
Möglichkeiten und vor allem der Bedarf, dieses Wissen durch den Einsatz von ERP-Systemen in der Lehre transparent zu machen, werden in der Literatur zahlreich diskutiert (z. B. Antonucci et al. 2004; Boyle und Strong 2006; Fedorowicz et al. 2004; Hawking et al. 2004; Peslak 2005; Stewart et al. 2000). Dabei wird klar, dass ERP-Systeme ein wichtiger Bestandteil der Curricula der Hochschulen in informationssystembezogenen Fächern sind bzw. seien sollten, dieses jedoch nicht trivial ist (Noguera und Watson 1999, S. 808).

Eines der Ziele von ERP-Systemen im Lehreinsatz ist die Vorbereitung der Studierenden auf das spätere Berufsleben, indem sie zumindest einen Einblick in ERP-Systeme erhalten. Ein weiteres Ziel wird durch die ERP-Hersteller vorangetrieben (insbesondere mit der Bereitstellung ihrer Systeme für die Lehre): Die Studierenden sollen mit ihren Produkten so früh wie möglich in Kontakt kommen, da die späteren Absolventen damit arbeiten und gegebenenfalls Positionen im Unternehmen besetzen könnten, die einen Einfluss auf die bereits angedeuteten Investitionsentscheidungen haben. Um dies zu erfüllen, ist es notwendig, dass Hochschulen entsprechende Systeme, Prozesse und somit passende Kurse anbieten (Brehm et al. 2009, S. 220; Fedorowicz et al. 2004, S. 235-236).

Jedoch gibt es dafür keinen standardisierten Ansatz. Die Systemwahl und die Anzahl der Systeme sowie der Aufbau und die Anzahl der ERP-Kurse divergieren von Hochschule zu Hochschule (Seethamraju 2007, S. 69). Im Gegensatz dazu ist festzustellen, dass, obwohl sich der ERP-Markt sehr heterogen darstellt, die Vielfalt der an Hochschulen vertretenen Systeme und Hersteller recht gering ist. Es dominieren vor allem einige große Hersteller den Einsatz in der Lehre. Zu nennen ist hier insbesondere der Hersteller SAP, der durch den Aufbau seines UniversityAlliance-Programms in zahlreichen Hochschulen vertreten ist. Mit mehr als 400 Partnerhochschulen in diesem Programm ist SAP damit das wohl am stärksten verbreitete System in der Lehre (Hawking et al. 2004, S. 328; Pellerin und Hadaya 2008, S. 65-66). Auch ist es durchaus für Studierende sinnvoll, sich mit diesem System zumindest ansatzweise im Studium auseinanderzusetzen, da aufgrund der hohen Marktanteile von SAP (siehe hierzu Konradin 2009, S. 75ff) die Wahrscheinlichkeit sehr hoch ist, dass sie mit SAP-Systemen in ihrem Berufsleben arbeiten werden.

Allerdings ist eine diversifiziertere Einbindung von ERP-Systemen vor allem aus dem Bereich der KMU ratsam. Diese Forderung resultiert nicht nur aus dem hohen Anteil dieser Unternehmen an der Gesamtunternehmenszahl - beispielsweise sind in Deutschland 99,5\% aller Unternehmen KMUs und beschäftigen über 60\% aller Arbeitnehmer (Schmiemann 2008, S. 3) - wodurch hier die Wahrscheinlichkeit für den späteren Absolventen auch recht hoch ist, in diesem Bereich mit ERP-Systemen in Kontakt zu kommen (sei es beispielsweise als Anwender oder als Mitarbeiter eines Beratungsunternehmens). Auch das Argument, den Studierenden mehr als nur ein bis zwei große Systeme zu zeigen, um einen Marktüberblick zu gewährleisten, unterstützt diese Forderung. Damit würden zusätzlich die Unterschiede von KMUs im Vergleich zu Großunternehmen vermittelt, die sich in den 
entsprechenden Ausprägungen dieser Systeme wiederspiegeln (Winkelmann und Klose 2008, S. 1-2). Weiterhin wird durch den Einsatz verschiedener ERP-Systeme in der Lehre eine Sensibilisierung der Studierenden für unterschiedliche funktionale Ansätze, Prozessunterstützungen sowie Oberflächenergonomie und Architekturkonzepte erreicht. ERP-Systeme und deren Konzepte können dabei auch ohne direkten Systembezug theoretisch erläutert werden. Jedoch werden die Lernerfahrung und das Verständnis durch den Einsatz von realen Systemen viel stärker gefördert (Watson und Schneider 1999, S. 4).

An diesem Punkt knüpft das von Winkelmann und Matzner (2009) beschriebene Seminar an. In dem darin vorgestellten Kurs wird ein problemorientierter oder auch lerner-zentrierter Lernansatz beschrieben (Saulnier et al. 2008; Stewart et al. 2000). Mit Fallstudien arbeiten sich die Studierenden in Kleingruppen selbstständig in verschiedene ERP-Systeme ein und präsentieren schlussendlich ihre Erkenntnisse beispielsweise live am jeweiligen System. Dadurch können die Teilnehmer des Seminars durch die Untersuchung von verschiedenen Systemen ihre Kenntnisse über ERP-Systeme (z. B. Funktionsumfang, Oberflächengestaltung, Benutzerfreundlichkeit, etc.) vertiefen und ausbauen.

Dieser Artikel greift das Seminarmodell von Winkelmann und Matzner (2009) auf und modifiziert und erweitert das Konzept, um es parallel an drei verschiedenen Universitätsstandorten anwenden zu können. Dabei ist anzumerken, dass die Studierendengruppen sowohl bei den Studiengängen (Bachelor, Diplom und Master) als auch bei der Anzahl sehr heterogen waren. Es wird daher auch beleuchtet, welchen Einfluss die Anzahl der Teammitglieder auf den Erfolg des Seminars hat und wie sich die Fachsemesteranzahl der Teilnehmer auswirkt.

Dabei steht nicht die detaillierte empirische Auswertung im Vordergrund, die aufgrund der geringen Seminargrößen ohnehin statistisch nicht relevant ist, sondern vielmehr die Darlegung gesammelter Erfahrungen mit dem Ziel, dieses Wissen auch für andere Universitäten und Hochschulen zur Verfügung zu stellen. Um die Fragen zu beantworten, ist der vorliegende Artikel wie folgt aufgebaut. Im zweiten Kapitel wird das Vorgehensmodell zum Aufbau und zur Durchführung des Seminars detailliert beschrieben, um damit einen Grundstein für eine mögliche Adaption an anderen Hochschulen zu legen. Im dritten Kapitel erfolgt anschließend die Bewertung der Evaluation des Seminars, wodurch direkte Rückschlüsse auf die aufgestellten Fragen gezogen werden können. Abschließend endet der Beitrag mit einer kurzen Zusammenfassung dieses Seminarkonzeptes.

\section{Vorgehensmodell und Beschreibung des Kurses}

In Anlehnung an das Vorgehensmodell von Winkelmann und Matzner (2009) wurde eine mehrstufige Prozedur zum Aufbau des Seminars und zur Auswahl der ERP-Systeme gewählt (vgl. Abbildung 1). Dazu wurde im ersten Schritt das zu behandelnde Thema der Studierenden abgegrenzt (z. B. Betrachtung der Produktion) und - falls anwendbar - ein domänensperifisches Framework gewählt, innerhalb 
dessen die Teilnehmer die gestellten Aufgaben bearbeiten mussten (Schritt 1). Darauf folgte die Auswahl der geeigneten ERP-Systeme. Dazu musste sich der Seminarleiter zuerst einen Überblick über die aktuelle Situation auf dem ERP-Markt verschaffen (Schritt 2), so dass er diejenigen Anbieter und Systeme auswäblen konnte, die für die zuvor definierte Aufgabenstellung den größten Lernerfolg versprechen (Schritt 3a). Problemorientiertes Lernen hat sich dabei als erfolgreiches Konzept für die Lehre von Informationssystemen etabliert (Stewart et al. 2000). Demnach verwendet das Szenario real existierende Prozesse aus Unternehmen, die den Studierenden als Ausgangspunkt für ihre Evaluation dienen (Schritt 3b). Am Ende des Semesters erfolgte nach der Analyse der jeweiligen Systeme (Schritt 4) als Abschluss des Kurses eine Präsentation der Ergebnisse (Schritt 5). Die Schritte 1 bis 3b lagen im Aufgabenbereich des Dozenten, während die Analyse und Präsentation den teilnehmenden Studierenden oblag.

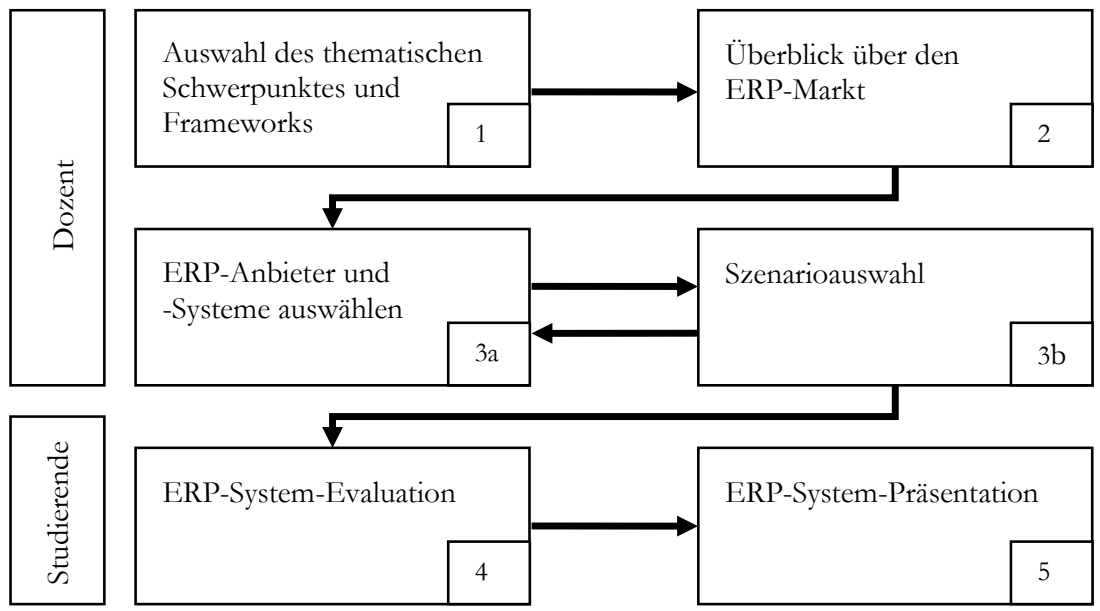

Abbildung 1: Vorgehensmodell nach Winkelmann und Matzner (2009, S. 3)

Das Vorgehensmodell wurde im Vergleich zu Winkelmann und Matzner (2009) nicht an einer Hochschule, sondern auf drei parallel stattfindende Seminare an den drei deutschen Universitäten Münster, Dresden und Koblenz-Landau angewandt. Dadurch konnte sichergestellt werden, dass sowohl die Vorgehensweise bei der Umsetzung des Modells als auch die von den Studierenden erzeugten Analysen an allen drei Standorten vergleichbar sind. Die Vergleichbarkeit der Ergebnisse war zudem eine zwingende Voraussetzung, um die Beantwortung der zu Beginn aufgestellten Fragen zu gewährleisten, wobei die Anzahl der Studierenden und die Fachsemesterzahl variierten.

- Anzahl der Studierenden: Während in Münster drei bis vier und in Dresden vier bis sechs Studierende pro System zur Verfügung standen, waren es in Koblenz- 
Landau bis auf eine Gruppe jeweils Zweierteams. Ein Team bestand nur aus einem Studierenden.

- Studiengang der Studierenden: In Münster befanden sich alle teilnehmenden Studierenden im Masterstudiengang Wirtschaftsinformatik (7.-9. Fachsemester), in Dresden bestanden die Teams sowohl aus Diplom- als auch Masterstudierenden (7. Fachsemester und höher). Die Teilnehmer in Koblenz waren abweichend von der Forderung nach Masterstudierenden im ursprünglichen Versuch Bachelorstudierende im fünften bzw. sechsten Semester.

\subsection{Auswahl des thematischen Schwerpunktes und Frameworks}

Heutzutage bieten bereits kleine und mittelgroße ERP-Anbieter komplexe und umfangreiche Funktionalitäten, um ein möglichst breites Spektrum für potentielle Kunden abdecken zu können. Aufgrund des Umfangs eines realen Einführungsprojekts und der Berücksichtigung des Ausbildungsstandes der teilnehmenden Studierenden ist eine Eingrenzung der abzuarbeitenden Prozesse daher sinnvoll.

Technische und domänenspezifische Frameworks sind sowohl für die Auswahl von geeigneten ERP-Systemen als auch als Leitlinie für die spätere Auswertung der studentischen Teilnehmer geeignet. Technische Frameworks wie z. B. das ARISHaus (Scheer et al. 2004) oder das Framework von Zachman (1987) bieten formale und hoch strukturierte Ansätze zur Gestaltung eines Unternehmens oder seiner zugrunde liegenden IT-Infrastruktur. Domänenspezifische Frameworks liefern strukturierte Daten und Informationen über eine Domäne.

In dem Szenario wurde, wie von Winkelmann und Matzner (2009, S. 3-4) vorgeschlagen, das Handels-H-Framework präferiert (Becker et al. 2001; Klaus et al. 2000), welches sowohl in der Lehre als auch in der Praxis international seinen Einsatz findet. Es dient als Referenzmodell für Handelsunternehmen, welches zwischen Funktionen, Daten und Prozessen unterscheidet. Das Handels-H eignet sich insbesondere für diese Seminarform, da es die Betrachtung von TeilFunktionalitäten zulässt, wodurch je nach Aufgabenstellung nur spezifische Funktionen verwendet werden können. Außerdem werden die Studierenden mit BestPractice Prozessen und den darunter liegenden Datenmodellen vertraut gemacht. Dadurch können sie auf Daten-, Funktions- und Prozessebene unterschiedliche Ansätze der Anforderungsanalyse für ERP-Systeme kennenlernen.

\section{2 Überblick über den ERP-Markt}

Es gibt zahlreiche Möglichkeiten, sich einen Überblick über den ERP-Markt zu verschaffen, allerdings sind viele der sogenannten Marktübersichten meist auf die großen Anbieter (SAP, Microsoft, Oracle usw.) fokussiert, so dass viele kleinere Systeme aus der Betrachtung fallen. Winkelmann und Matzner (2009, S. 4-5) schlagen mehrere Methoden vor, mit denen sich das Spektrum möglicher ERP-Anbieter für ein ERP-Seminar erweitern lässt: Persönliche Meetings auf Konferenzen oder 
Messen (z. B. CeBIT), Artikel über ERP-Systeme in Fachzeitschriften (z. B. ERPManager) und Marktübersichtsstudien bzw. Plattformen zur Software Evaluation (z. B. IT-Matchmaker). Diese Methoden bieten alle einen schnellen und intuitiven Zugang zum ERP-Markt.

In der vorliegenden Untersuchung wurde zusätzlich eine weitere Quelle genutzt: Unternehmensfallstudien. Sie zeichnen sich durch eine wissenschaftlich fundierte Forschungsmethodik aus, die bei gleichbleibender Struktur die Vergleichbarkeit von verschiedenen Systemen ermöglicht (Beispiel: eXperienceForschungsmethodik (Schubert und Wölfle 2007)). Nachteile dieser Methode ergeben sich zum Einen aus den unterschiedlichen Szenarien, die im Fokus der Fallstudien stehen können, zum Anderen wird typischerweise immer nur ein System in einer Fallstudie betrachtet. Dennoch war diese Methode eine sinnvolle Ergänzung zu den postulierten Ansätzen, da sie einen strukturierten Vergleich mehrerer ERPSysteme ermöglicht.

\subsection{ERP-Anbieter und -Systeme auswählen}

Die Auswahl der geeigneten Systeme erfolgte nach den Kriterien

- Unternehmensgröße/Kundenbasis: Bekanntheitsgrad und Wichtigkeit des Anbieters im Markt

- Funktionalität: Umfang der funktionalen Unterstützung des erstellten Szenarios

- Alter: Erfahrung des Anbieters auf dem Markt

- Ergonomie: Effizienz und Effektivität der Benutzersteuerung

- Zugriff: Systemnutzung mit vertretbarem Aufwand - entweder durch eigene Installation durch Studierende oder durch Fernzugriff.

Alle ausgewählten Systeme boten Funktionalitäten für Handel, Produktion und Lagerwirtschaft. Die Anbieter wurden um einen Remote-Zugriff auf ihr System gebeten, dem drei der fünf Hersteller nachkamen. Die beiden anderen Systeme wurden vor Ort auf universitären Rechnern zur Verfügung gestellt. Die entsprechenden Lizenzen und Vollversionen der ERP-Systeme wurden kostenfrei für den Zeitraum des Seminars freigegeben. Im Vorfeld wurde außerdem das Szenario an jeden Hersteller übermittelt, so dass der Anbieter zum einen über Umfang und Ziel des Seminars transparent informiert war, zum anderen konnten bestimmte Einstellungen an den Systemen (z. B. Anzahl der gleichzeitigen User-Zugriffe) vorgenommen werden. Insgesamt wurden sieben Systeme von fünf Herstellern evaluiert. Als Ansprechpartner dienten Mitarbeiter aus der Marketing-Abteilung oder der Geschäftsführung (Kontaktaufnahme) und Mitarbeiter aus dem Support (Durchführung des Seminars).

\subsection{Szenarioauswahl}

Die Auswahl der passenden ERP-Systeme und des Szenarios für die Studierenden bedingen einander. Es ist nicht zielführend, auf Produktion spezialisierte Anbieter 
um Ihre Mitarbeit zu bitten, wenn das Szenario nur Handelsfunktionen enthält. Umgekehrt ist es nicht sinnvoll, Prozesse aus der Produktion in Systemen evaluieren zu lassen, die keinerlei Funktionalität diesbezüglich enthalten.

Das Szenario enthielt entsprechend des Handels-H-Frameworks allgemeine und spezielle Handelsprozesse, die von den Studierenden bearbeitet wurden. Zusätzlich wurde dem Szenario ein allgemeiner Produktionsprozess hinzugefügt, der die Montage eines Produktes aus Einzelteilen vorsah. Ursprünglich waren nur Handelsprozesse als Aufgabenbereich für die Studierenden vorgesehen. Während der Auswahlphase der möglichen Anbieter hat sich jedoch gezeigt, dass alle ERPSysteme laut Herstellerangabe auch Funktionalitäten im Produktionsbereich anboten. Aus diesem Grund wurde ein allgemein gehaltener Produktionsprozess mit in die Fallstudie einbezogen, um den Evaluationsbereich der Systeme zu erweitern. Aus Platzgründen kann die 13seitige Fallstudie nicht ausführlich erläutert werden. Sie kann jedoch vom Erstautor in Deutsch und Englisch angefordert werden.

\subsection{Seminardurchführung und ERP-System-Evaluation}

$\mathrm{Zu}$ Beginn des Seminars wurden den Studierenden in einer Kickoff-Veranstaltung die organisatorischen Rahmenbedingungen des Seminars erläutert, eine inhaltliche Vorstellung des zu bearbeitenden Szenarios gegeben und die zu leistenden Aufgaben definiert. Während des Seminars arbeiteten die Teilnehmer die vorgegebenen Prozesse in Kleingruppen im jeweiligen ERP-System ab und erstellten am Ende des Semesters eine schriftliche Evaluation des jeweiligen ERP-Systems. Dazu wurden diejenigen Prozesse modelliert, die mit der Funktionalität des ERP-Systems umsetzbar waren. Zusätzlich wurden noch für das Szenario wichtige Datenmodelle erstellt (z. B. für die Lagerorganisation).

Die Betreuungsleistung der Dozenten erstreckte sich dabei in der Regel auf individuell vereinbarte Termine, in denen die Teams Fragen zu technischen oder inhaltlichen Problemen stellen konnten.

Neben der grafischen Aufbereitung der Prozesse und der Datenmodelle waren weiterhin eine Zusammenfassung der technischen Grundlagen des zugewiesenen Systems sowie ein abschließendes Fazit erforderlich, das Vor- und Nachteile der Lösung beleuchtet. Dabei war es den Studierenden überlassen, welchen Schwerpunkt sie bei der Evaluation ihres Systems wählen. Hervorzuheben ist, dass es keine Schulung durch die ERP-Anbieter gab, sondern die Einarbeitung der Studierenden nach der Bereitstellung der Systeme selbstständig geschah. Der Kontakt zum Hersteller war immer nur dann notwendig, wenn ein technisches Problem eine Weiterbearbeitung des Szenarios verhinderte (z. B. fehlende Zugriffsrechte, Auslaufen der Lizenz). 


\subsection{ERP-System-Präsentationen}

Die Präsentation in einer zweitägigen Blockveranstaltung hat sich als praktikabel herausgestellt, da die ERP-Systeme und ihre Funktionalität in einem kurzen Zeitraum verdichtet dargestellt wurden und so eine Vergleichbarkeit der Software erleichtert wird. Jedes Team hat dabei 60 Minuten Präsentationszeit und zusätzlich 30 Minuten für eine Diskussion und offene Fragen.

Die meisten der studentischen Teams hatten sich für eine Präsentation ihrer Ergebnisse zusammen mit einer Live-Präsentation "ihres" ERP-Systems entschieden. Sämtliche Teams (im Besonderen diejenigen ohne eine Vor-Ort-Installation) konnten die Demonstration dank der stabilen Verbindungen zu ihrem jeweiligen System durchführen. Dadurch ließen sich die vorgestellten Prozesse direkt im System nachvollziehen.

An den Präsentationsterminen herrschte Anwesenheitspflicht für alle Studierende. Dadurch wurde sichergestellt, dass der Lerninhalt und Lernerfolg nicht auf ein ERP-System beschränkt blieb. Zudem wurde bei der Durchführung des Seminars pro System ein prämierter Wettbewerb zwischen den drei Universitäten für die jeweils beste Systemdurchdringung und -präsentation ausgelobt.

\section{Bewertung des Seminars}

\subsection{Perspektive der Studierenden}

Aufgrund der Tatsache, dass eine Auswertung von Seminaren bzw. allgemein von Lehrveranstaltungen hohe Wichtigkeit zur Verbesserung der Lehrkonzepte besitzt (Seethamraju 2007, S. 80), wurden zur Evaluation des Seminars an allen drei Universitäten nach Abschluss der Systempräsentationen Fragebögen an die Studierenden verteilt, welche anonym ausgefüllt wurden. Dies diente dazu, einerseits mögliche Schwächen und Verbesserungsmöglichkeiten bei Durchführung, Aufgabenstellung und Betreuung von Seiten der Hochschule und von Seiten der ERP-Hersteller aufzudecken. Andererseits sollten damit auch die positiven Aspekte hervorgehoben werden, die bei einem erneuten Durchlauf des Seminars beibehalten werden sollten. Der Fragebogen bestand aus insgesamt 23 Fragen, wobei dieser sowohl Skalenbewertungen (Noten 1-5), Ja/Nein-Fragen als auch Freitextantworten beinhaltete. Zusätzlich wurden mit jedem Team separate Feedbackgespräche geführt, um weitere Anregungen der Studierenden zu erfassen.

Bei Auswertung der Fragebögen und Gespräche hat sich gezeigt, dass ausnahmslos alle Teilnehmer angaben, einen hohen Wissenszuwachs bezüglich fachlicher, methodischer, sozialer und technischer Aspekte erworben zu haben. Auch der Nutzen der Fallstudie wurde als sehr hoch eingestuft.

Bei der Aufwandsbewertung des Seminars war vor allem in Koblenz-Landau zu erkennen, dass hier der Aufwand im Gegensatz zu Dresden und Münster als deutlich höher eingestuft wurde, wohingegen die Studierenden in Dresden das 
Seminar vom Aufwand her am unkritischsten betrachteten. Bereits zu Beginn des Seminars zeigte sich in Koblenz-Landau, dass eine regelmäßig durchgeführte Übungsstruktur den Bachelorstudierenden eine deutliche Hilfestellung zur Unterstützung der individuellen Projektorganisation und Präsentationstechniken leisten konnte. Dies wurde von den Koblenzer Teams sehr begrüßt, während in Dresden und Münster eine konstante Betreuungsleistung nicht gefordert wurde. Eine weitere Erklärung lässt sich in der Anzahl der Studierenden pro Team bzw. ERP-System finden. Während, wie bereits in Kapitel 2 erwähnt, in Koblenz die Teams meistens nur aus zwei Studierenden bestanden, war die Mitgliederzahl in Dresden im Schnitt bei fünf Studierenden, wodurch die Arbeitspakete besser aufzuteilen waren und das Wissen über das System schneller erarbeitet werden konnte. Dies lässt sich auch sehr deutlich durch die Freitextantworten der Fragebögen erfassen. Dabei waren die Teams in Koblenz einstimmig der Meinung, dass der Umfang der Fallstudie reduziert werden müsse, während dies in Münster und Dresden nicht als notwendig erachtet wurde.

Die Studierenden konnten sich standortübergreifend per Email austauschen. Dadurch konnten bei Problemen entsprechende Informationen von den Teams der anderen Standorte erfragt werden. Dieses wurde sehr positiv aufgenommen. Es entstand ein reger Austausch über die drei Universitäten hinweg. Auch der Wettbewerb zwischen den Universitäten erzeugte nicht nur einen internen Anreiz für ein gutes Projektergebnis, sondern diente zusätzlich zur Anregung der standortübergreifenden Kommunikation der Studierenden. Dadurch wurde, immer auf die Teams desselben Systems bezogen, ein fachlicher und zwischenmenschlicher Austausch von Informationen gefördert, was wiederum zur Verbesserung der SoftSkills der Studierenden diente.

\subsection{Perspektive der ERP-System-Hersteller}

Da dieser Kurs die Fortführung bzw. Ausweitung des von Winkelmann und Matzner (2009) beschriebenen Modells ist, bestand zu einigen ERP-Herstellern schon im Vorfeld Kontakt bzw. hatten diese ihre Systeme schon bei vorherigen Durchläufen zur Verfügung gestellt. Einer der Hersteller betreibt zudem ein Programm ähnlich dem University-Alliance-Programm von SAP. Zwei der Hersteller waren jedoch noch nicht mit diesem Seminar vertraut und hatten wegen möglicher Konkurrenzspionage Bedenken. Um diese Zweifel auszuräumen, wurde vereinbart, dass Mitarbeiter der Hersteller auf Wunsch gerne den Präsentationen ihrer eigenen Systeme durch die jeweiligen Teams beiwohnen konnten, jedoch nicht an den Präsentationen der Wettbewerber. Dieses Angebot wurde im Rahmen des Seminars von einem Hersteller wahrgenommen.

Weiterhin bestand die Möglichkeit von Seiten der Hersteller entsprechend den Zugang zu ihren Systemen zu beschränken. Während zwei Hersteller Vollversionen mit 1-Jahres-Lizenzen zur Verfügung stellten, wurde der Zugang zu den anderen drei Systemen durch eine IP-bezogene Remote-Verbindung ermöglicht, wo- 
durch die Teams nur von speziellen Rechnern innerhalb der Universitäten auf die ERP-Systeme zugreifen konnten. Alle fünf Hersteller zeigten sich mit dem Ablauf und den Ergebnissen des Seminars sehr zufrieden und waren an den Auswertungen / Berichten der Studierenden zu ihren Systemen sehr interessiert. Einigen Studierenden wurde sogar die Möglichkeit eines Praktikums offeriert.

\section{Zusammenfassung}

Die Idee des originären Kurses, eine Ergänzung zum Einsatz von ERP-Systemen (für Großunternehmen) in der Lehre zu schaffen, wurde durch das beschriebene Seminar aufgegriffen und fortgeführt. Durch die Ausweitung des Seminars auf drei Universitäten bot sich die Möglichkeit, verschiedene Rahmenbedingungen anzupassen, um damit deren Einfluss auf den Erfolg des Seminars und auf die Wahrnehmung von Seiten der Studierenden zu messen. Dabei hat sich gezeigt, dass eine zu geringe Gruppengröße (siehe Beispiel Koblenz) für dieses Seminar nicht förderlich ist, da somit der Aufwand von den Studierenden als sehr hoch eingestuft und das Seminar eher als Belastung anstatt als Wissensbereicherung angesehen wird. Somit zeigt sich eine deutliche Abhängigkeit zwischen Seminarerfolg, -aufwand und Anzahl der Teammitglieder. Dieses ließ sich aufgrund der geringen Fallzahlen nicht empirisch validieren. Bei den Feedbackgesprächen stellten sich 3er-Teams für das gleichzeitige Arbeiten am System bzw. größere Teams für das arbeitsteilige Lösen der Aufgaben als durchaus sinnvoll heraus. Bezüglich Semesteranzahl kann abschließend festgestellt werden, dass auch die Art der Studiengänge, oder genauer gesagt, die Anzahl der von den Studierenden absolvierten Fachsemester einen Einfluss auf das Seminar haben. In den Feedbackgesprächen im Anschluss an die Systempräsentationen konnte ermittelt werden, dass vor allem Studierende höherer Semester das Seminar als „passend“ ansahen, während Studierende niedrigerer Semester erheblich mehr Aufwand in noch nicht vollständig entwickelte Fähigkeiten zur Projektorganisation und Präsentationstechniken investieren mussten und daher das Seminar im Vergleich zum erlangten Wissenszuwachs als zu aufwandsintensiv ansahen. Dies kann aber, wie in Koblenz erfolgt, durch eine intensive Betreuung von Seiten der Dozenten kompensiert werden.

Als abschließendes Fazit ist somit festzuhalten, dass das Seminar sowohl für die Studierenden als auch für die Dozenten eine gute Möglichkeit eröffnet, Einblicke in verschiedene ERP-Systeme zu erhalten und so ihre Kenntnisse und die Wahrnehmung bezüglich der Vielfalt der ERP-Systeme zu erweitern und zu schärfen.

\section{Literatur}

Antonucci YL, Corbitt G, Stewart G, Harris AL (2004) Enterprise systems education: Where are we? Where are we going? Journal of Information Systems Education 15(3):227-234. 
Barker T, Frolick MN (2003) ERP implementation failure: A case study. Information Systems Management 20(4):43-49.

Becker J, Uhr W, Vering O, Ehlers L, Kosilek E, Lohse M, Neumann S (2001) Retail information systems based on SAP products. Springer, Berlin.

Becker J, Vering O, Winkelmann A (2007) Software-Auswahl und -Einführung in Industrie und Handel. Vorgehen bei und Erfahrungen mit ERP- und Warenwirtschaftssystemen. Springer, Berlin, Heidelberg.

Boyle TA, Strong SE (2006) Skill requirements of ERP graduates. Journal of Information Systems Education 17(4):403-412.

Brehm N, Haak L, Peters D (2009) Using FERP Systems to introduce web servicebased ERP Systems in higher education. In: Abramowicz W, Flejter D (Hrsg) Business Information Systems Workshops: BIS 2009 International Workshops, Poznan, Poland, April 27-29, 2009, Revised Papers: 37. Springer, Berlin.

Fedorowicz J, Gelinas UJJ, Usoff C, Hachey G (2004) Twelve tips for successfully integrating enterprise systems across the curriculum. Journal of Information Systems Education 15(3):235-244.

Hawking P, McCarthy B, Stein A (2004) Second wave ERP education. Journal of Information Systems Education 15(3):327-332.

Hsu K, Sylvestre J, Sayed EN (2006) Avoiding ERP Pitfalls. The Journal of Corporate Accounting \& Finance 17(4):67-74.

Klaus H, Rosemann M, Gable GG (2000) What is ERP?. Information Systems Frontier 2(2):141-162.

Konradin Business GmbH (2009) Konradin ERP-Studie 2009: Einsatz von ERPLösungen in der Industrie. Konradin Mediengruppe, Leinfelden-Echterdingen.

Noguera JH, Watson EF (1999) Effectiveness of using an enterprise system to teach process-centered concepts in business education. In: Proceedings of the 5th annual Americas Conference on Information Systems, Milwaukee.

Pellerin R, Hadaya P (2008) Proposing a new framework and an innovative approach to teaching reengineering and ERP implementation concepts. Journal of Information Systems Education 19(1):65-73.

Peslak AR (2005) A twelve-step, multiple course approach to teaching enterprise resource planning. Journal of Information Systems Education 16(2):147-155.

Saulnier BM, Landry JP, Longenecker HEJ, Wagner TA (2008) From teaching to learning: Learner-centered teaching and assessment in information systems education. Journal of Information Systems Education 19(2):169-174. 
Scheer AW, Abolhassan F, Jost W, Kirchmer M (2004) Business process automation: ARIS in practice. Springer, Berlin.

Schmiemann M (2008) Unternehmen nach Größenklassen: Überblick über KMU in der EU. eurostat - Statistik kurz zusammengefasst 31(2008).

Schubert P, Wölfle R (2007) The eXperience methodology for writing IS case studies. In: Proceedings of the Thirteenth American Conference on Information Systems, Keystone.

Seethamraju R (2007) Enterprise systems software in business school curriculum Evaluation of design and delivery. Journal of Information Systems Education 18(1):69-83.

Stewart G, Rosemann M, Hawking P (2000) Collaborative ERP curriculum developing using industry process models. In: Proceedings of the 6th annual Americas Conference on Information Systems, Long Beach.

Venkatesh V (2008) “One-Size-Does-Not-Fit-All”: Teaching MBA students different ERP implementation strategies. Journal of Information Systems Education 19(2):141-146.

Watson EE, Schneider H (1999) Using ERP systems in education.

Communications of the AIS 1(2).

Winkelmann A, Knackstedt R, Vering O (2007) Anpassung und Entwicklung von Warenwirtschaftssystemen - eine explorative Untersuchung. Handelsstudie Nr. 3, Münster. http://www.wi.uni-

muenster.de/udoo/downloads/publications/1975.pdf. Abruf am 2009-09-10.

Winkelmann A, Klose K (2008) Experiences while selecting, adapting and implementing ERP systems in SMEs: a case study. In: Proceedings of the 14 th Americas Conference on Information Systems, Toronto.

Winkelmann A, Matzner M (2009) Teaching medium sized ERP systems - a problem-based learning approach. In: Papadopoulos GA, Wojtkowski W, Wojtkowski WG, Wrycza S, Zupancic, J. (Hrsg) Information Systems Development: Towards a Service Provision Society. Springer, New York.

Zachman JA (1987) A framework for information systems architecture. IBM Systems Journal 36(3):277-293. 